\title{
Nicotine promotes cell proliferation and induces resistance to cisplatin by $\alpha 7$ nicotinic acetylcholine receptor-mediated activation in Raw264.7 and El4 cells
}

\author{
YAN YAN WANG ${ }^{*}$, YAO LIU*, XIAO YAN NI ${ }^{*}$, ZHEN HUAN BAI, \\ QIONG YUN CHEN, YE ZHANG and FENG GUANG GAO
}

\author{
Basic Medicine Science, Medical College, Xiamen University, Xiamen, Fujian 361005, P.R. China
}

Received November 26, 2013; Accepted December 19, 2013

DOI: $10.3892 /$ or.2013.2962

\begin{abstract}
Although nicotine is a risk factor for carcinogenesis and atherosclerosis, epidemiological data indicate that nicotine has therapeutic benefits in treating Alzheimer's disease. Our previous studies also showed that nicotine-treated dendritic cells have potential antitumor effects. Hence, the precise effects of nicotine on the biological characterizations of cells are controversial. The aim of the present study was to assess the roles of $\alpha 7$ nicotinic acetylcholine receptors (nAChRs), Erk1/2-p38-JNK and PI3K-Akt pathway in nicotine-mediated proliferation and anti-apoptosis effects. The results firstly showed that nicotine treatment clearly augmented cell viability and upregulated PCNA expression in both Raw264.7 and E14 cells. Meanwhile, nicotine afforded protection against cisplatin-induced toxicity through inhibiting caspase-3 activation and upregulating anti-apoptotic protein expression. Further exploration demonstrated that nicotine efficiently abolished cisplatin-promoted mitochondria translocation of Bax and the release of cytochrome $c$. The pretreatment of $\alpha$-bungarotoxin and tubocurarine chloride significantly attenuated nicotineaugmented cell viability, abolished caspase- 3 activation and $\alpha 7 \mathrm{nAChR}$ upregulation. Both Erk-JNK-p38 and PI3K-Akt signaling pathways could be activated by nicotine treatment in Raw264.7 and E14 cells. Notably, when Erk-JNK and PI3K-Akt activities were inhibited, nicotine-augmented cell proliferation and anti-apoptotic effects were abolished accordingly. The results presented here indicate that nicotine could achieve $\alpha 7 \mathrm{nAChR}$-mediated proliferation and anti-apoptotic
\end{abstract}

Correspondence to: Professor Feng Guang Gao or Professor Ye Zhang, Basic Medicine Science, Medical College, Xiamen University, Xiamen, Fujian 361005, P.R. China

E-mail: gfengguang@xmu.edu.cn

E-mail: zhangye@xmu.edu.cn

${ }^{*}$ Contributed equally

Key words: nicotine, $\alpha 7$ nicotinic acetylcholine receptors, MAPK, proliferation, anti-apoptosis, Akt effects by activating Erk-JNK and PI3K-Akt pathways respectively, providing potential therapeutic molecules to deal with smoking-associated human diseases.

\section{Introduction}

Cigarette smoking, which causes lung and other types of cancer, is also a risk factor of both established tumor metastasis and increases overall mortality in cancer patients $(1,2)$. In addition, smokers have increased vulnerability to atherosclerosis and are predisposed to allergic airway diseases $(3,4)$. These studies suggest that by altering cell viability, smoking enables tumor cells and vascular endothelial cells to evade appropriate immune responses. Nicotine, a major component of cigarette smoke, is widely accepted as a risk factor for carcinogenesis and atherosclerosis (1). However, to date, little is known about how and to what extent nicotine contributes to the adverse effects of chronic tobacco use, apart from its psychoactive actions and addictive properties. Although nicotine could promote lung cancer development, reduce the efficacy of chemotherapeutic agents (5) and activate hypoxia-inducible factor-1 $\alpha$ expression (6), the fast synaptic transmission in key regions controlling behavior mediated by nicotine via nicotine acetylcholine receptors (nAChRs) was also reported (3), indicating that nicotine might be a survival agonist against various stresses inducing apoptosis (4). We previously studied the biological roles of nicotine and found that nicotine activates bone marrow-derived dendritic cells and nicotine-treated dendritic cells have potential antitumor effects (7-9). Hence, the exact effect of nicotine on cell survival has not been fully characterized and remains controversial.

nAChRs, which belong to a family of ionotropic receptors consisting of $\alpha$-subunits or a combination of $\alpha$ and $\beta$-subunits, are widely distributed throughout the central and peripheral nervous system (10). In addition, non-neuronal cells such as monocytes, endothelial cells and epithelial cells were also found to express nAChRs (11). Despite documented data showing that $\alpha 1, \alpha 3, \alpha 5 \mathrm{nAChRs}$ play important roles in regulating cell proliferation, apoptosis and facilitating tumor formation (12-14), $\alpha 7 \mathrm{nAChR}$, which is the main receptor of $\mathrm{nACh}$ in non-neuronal cells, has acquired more attention in recent years. For example, $\alpha 7 \mathrm{nAChR}$ was found to be 
involved in gastric and colon cancer migration $(15,16)$ and to also upregulate PPAR $\beta / \delta$ expression in human lung carcinoma (17). The fact that inhibition of $\alpha 7$-nicotinic receptor reduces tumorigenicity in A549 NSCLC xenografts (18) and facilitates lung cancer treatment (19) indicated that $\alpha 7 \mathrm{nAChR}$ plays important roles in tumor proliferation, angiogenesis and apoptosis (20-22). To date, however, the exact role of $\alpha 7 \mathrm{nAChR}$ in nicotine-mediated cell proliferation and anti-apoptotic effects has not been fully elucidated, and is important for potential tumor therapeutic target molecule discovery.

In the present study, to investigate the roles of $\alpha 7 \mathrm{nAChR}$ in nicotine-mediated cell proliferation and anti-apoptotic effects, Raw264.7 and El4 cells were treated with nicotine and cell proliferation was firstly determined by CCK- 8 assay and western blot analysis, respectively. Then, the roles of $\alpha 7 \mathrm{nAChR}$ in nicotine-augmented cell proliferation and antiapoptotic effects were further explored by pre-incubation of cells with $\alpha 7 \mathrm{nAChR}$ specific antagonist $\alpha$-bungarotoxin and broad spectrum nicotinic antagonist tubocurarine chloride. The effects of nicotine on cisplatin-induced mitochondria translocation of Bax and the release of cytochrome $c$ from mitochondria were investigated by mitochondria isolation and western blot analysis. Using kinase inhibitors, the roles of Erk-JNK-p38 and PI3K-Akt phosphorylation in nicotinemediated cell proliferation and anti-apoptosis effects were further investigated. The results showed that, firstly, nicotine treatment clearly augmented cell viability, upregulated Mcl-1 and Bcl-2 expression and decreased caspase-3 activation in Raw264.7 and El4 cells. Secondly, the pretreatment of $\alpha$-bungarotoxin and tubocurarine chloride efficiently attenuated nicotine-augmented cell proliferation, $\alpha 7 \mathrm{nAChR}$ upregulation and abolished the inhibitory effects of nicotine on cisplatin-induced caspase-3 activation. Further exploration demonstrated that nicotine efficiently abolished cisplatinpromoted mitochondria translocation of Bax and the release of cytochrome $c$. Notably, both Erk-JNK-p38 and PI3K-Akt signaling pathways could be activated by nicotine treatment in Raw264.7 and El4 cells. When Erk-JNK and PI3K-Akt activities were inhibited, nicotine-augmented cell proliferation ability and anti-apoptotic effects were reversed accordingly. The results presented here indicate that nicotine could achieve $\alpha 7$ nAChR-mediated proliferation and anti-apoptotic effects by activating the Erk-JNK and PI3K-Akt pathways respectively, providing potential molecules to deal with tobacco-associated human diseases.

\section{Materials and methods}

Reagents. Reagents were purchased from the following companies: nicotine, $\alpha$-bungarotoxin and tubocurarine chloride were obtained from Sigma-Aldrich (St. Louis, MO, USA). Cisplatin (DDP) was purchased from Calbiochem (San Diego, CA, USA). Annexin V/PI Apoptosis Detection kit was obtained from Promega (Madison, WI, USA). Cell Counting Kit-8 (CCK-8) was from Dojindo Laboratories (Kumamoto, Japan). p38 MAPK inhibitor SB203580, JNK MAPK inhibitor SP600125, Erk1/2 inhibitor U0126, PI3K inhibitor LY294002 and Akt inhibitor Wortmanin were from Cayman Chemical (Ann Arbor, MI, USA). Antibodies to $\beta$-actin, Cox IV, PCNA, $\alpha 7$ nAChR, Bcl-2, Mcl-1, Bax, cleaved caspase-3, cyto- chrome $c$, phospho-p38, phospho-Erk1/2, phospho-Mek1/2, phospho-c-Raf, phospho-Msk, phospho-p90Rsk, phosphoJNK were from Cell Signaling Technology (Beverly, MA, USA). Mitochondria isolation kit for cultured cells was from Thermo Fisher Scientific (Rockford, IL, USA). RPMI-1640 medium, Dulbecco's modified Eagle's medium (DMEM) and fetal bovine serum (FBS) were purchased from HyClone (Logan, UT, USA).

Cell line. The Raw264.7 and El4, macrophage and Tymphoma cell lines respectively, were obtained from Shanghai Cell Bank (Shanghai, China). Cells were cultured in DMEM with $10 \% \mathrm{FBS}$ at $37^{\circ} \mathrm{C}$ in $5 \% \mathrm{CO}_{2}$ and passed every $1-2$ days to maintain logarithmic growth. Cells were synchronized by serum starvation for at least $12 \mathrm{~h}$ before the treatment of nicotine for indicated periods or concentrations. To investigate the role of $\alpha 7 \mathrm{nAChR}$ in nicotine-mediated proliferation and anti-apoptotic effect, the cells were pretreated with $1 \mu \mathrm{g} / \mathrm{ml}$ $\alpha 7 \mathrm{nAChR}$ specific antagonist $\alpha$-bungarotoxin or broad spectrum nicotinic antagonist tubocurarine chloride prior to the indicated nicotine treatment.

Cell apoptosis assay. Cell apoptosis assay was determined by flow cytometry according to the method previously described (23). Briefly, 5x10 ${ }^{4}$ Raw 264.7 or El4 cells seeded in 24-well plates were pretreated with $10 \mu \mathrm{M}$ U0126, SB203580 or SP6001250 $1 \mathrm{~h}$ prior to $24-\mathrm{h} 10^{-7} \mathrm{M}$ nicotine stimulation and were further treated with $2 \mu \mathrm{g} / \mathrm{ml}$ cisplatin for $17 \mathrm{~h}$. Then, the cells were removed by trypsinization, rinsed with PBS and re-suspended in binding buffer containing Annexin V-FITC and propidium iodide (PI) for $20 \mathrm{~min}$ at room temperature. The samples were analyzed on FACSCalibur and data were analyzed with CellQuest software.

Cell proliferation assay. Cell proliferation assay was performed as previously described (24). Briefly, $5 \times 10^{4}$ El4 or Raw264.7 cells were inoculated in 96-well plate in $100 \mu \mathrm{l} /$ well medium in a humidified incubator $\left(37^{\circ} \mathrm{C}, 5 \% \mathrm{CO}_{2}\right)$. Then, the cells were treated with $10 \mu \mathrm{M}$ LY294002/Wortmanin or $1 \mu \mathrm{g} / \mathrm{ml}$ tubocurarine chloride/ $\alpha$-bungarotoxin $60 \mathrm{~min}$ prior to $10^{-7} \mathrm{~mol} / 1$ nicotine stimulation for indicated periods. CCK-8 solutions (10 $\mu \mathrm{l})$ were added to each well of the plate and OD450 value was detected with the wavelength of $450 \mathrm{~nm}$.

Mitochondria isolation. To explore the effect of nicotine on cisplatin-induced mitochondrial translocation of Bax and the release of cytochrome $c, 2 \times 10^{7}$ Raw264.7 cells were treated with $10^{-7} \mathrm{~mol} / \mathrm{l}$ nicotine for $8 \mathrm{~h}$ prior to cisplatin (1 $\mu \mathrm{g} / \mathrm{ml}$ ) treatment. The mitochondria isolation was performed according to the standard procedure. Briefly, cell pellet was harvested by $850 \mathrm{x}$ g centrifuging and vortexed at medium speed in $800 \mu$ l Mitochondria Isolation Reagent A. Then, $10 \mu 1$ Mitochondria Isolation Reagent B was added and incubated on ice for $5 \mathrm{~min}$ with further lysis by $800 \mu \mathrm{l}$ Mitochondria Isolation Reagent C. After 15 min 12,000 x g centrifugation, the pellet contained isolated mitochondria and the supernatant contained cytosol fraction. The Bax translocation and cytochrome $c$ release were determined by western blot analysis, respectively. $\beta$-actin and Cox IV were used as internal control. 
A

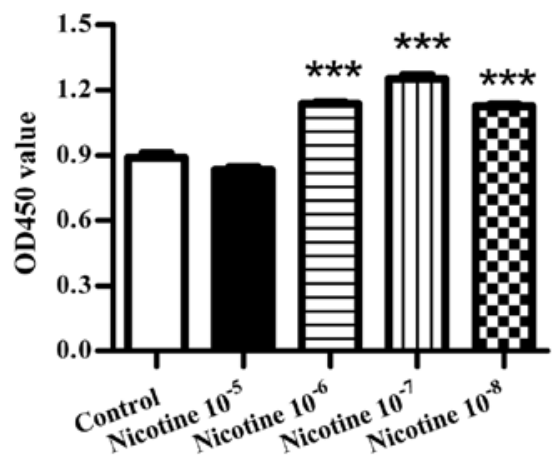

C

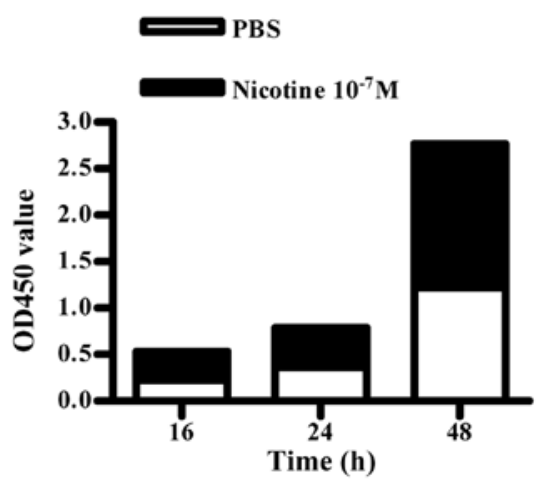

E

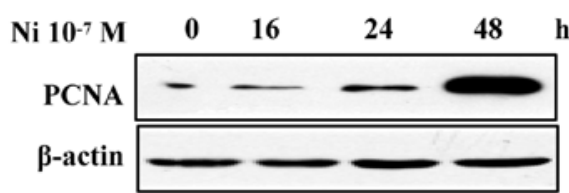

B

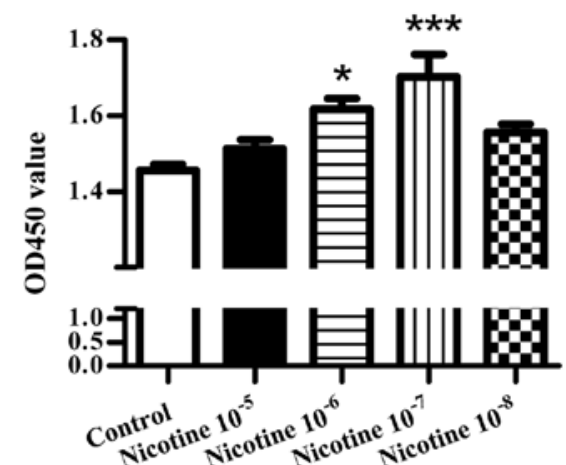

D

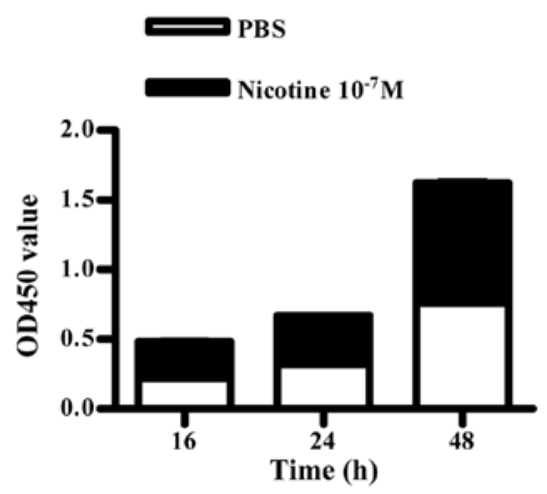

F

Figure 1. Nicotine treatment augments cell proliferation in Raw264.7 and El4 cells. (A, C and E) 3x10 4 Raw264.7 cells or (B, D and F) El4 cells were treated with the indicated concentrations of nicotine for $24 \mathrm{~h}$ (A and B) or $10^{-7} \mathrm{~mol} / 1$ nicotine for the indicated periods (C-F). Then, the cell viabilities of Raw264.7 (A and C) and El4 (B and D) were determined by CCK-8 assay. Data are given as mean \pm SEM, $n=4 .{ }^{* * *} \mathrm{P}<0.001$, ${ }^{*} \mathrm{P}<0.05$, one way ANOVA with post Newman-Keuls test. Meanwhile, the whole cellular protein of (E) Raw264.7 or (F) El4 stimulated with $10^{-7}$ mol/1 nicotine for $24 \mathrm{~h}$ was extracted and the effects of nicotine on PCNA expression were determined by western blot analysis. $\beta$-actin was used as internal control. A representative of three independent experiments is shown $(\mathrm{n}=3)$. $\mathrm{Ni}$, nicotine.

Western blot analysis. Proteins were obtained in lysis buffer as previously described (7). For analysis of PI3K-Akt, MAPK kinases phosphorylation and upregulation of Bcl-2, Mcl-1, PCNA, $\alpha 7 \mathrm{nAChR}$ induced by nicotine stimulation, $2 \times 10^{7}$ Raw264.7 or El4 cells were treated with $10^{-7} \mathrm{~mol} / \mathrm{l}$ nicotine stimulation for indicated periods. To explore the role of $\alpha 7 \mathrm{nAChR}$ in nicotine-augmented cell proliferation and antiapoptotic effect, cells were treated with $1 \mu \mathrm{g} / \mathrm{ml}$ tubocurarine chloride or $\alpha$-bungarotoxin $60 \mathrm{~min}$ prior to $24 \mathrm{~h} 10^{-7} \mathrm{~mol} / 1$ nicotine stimulation. Proteins were loaded onto SDS-PAGE gels for electrophoresis and then transferred onto PVDF membranes. After blocking in 5\% fat-free milk in TBST for $1.5 \mathrm{~h}$, the membranes were incubated with primary antibodies at $4^{\circ} \mathrm{C}$ overnight. Subsequently, the membranes were incubated with corresponding HRP-conjugated secondary antibodies at room temperature for $1.5 \mathrm{~h}$. After washing six times with TBST (for $10 \mathrm{~min}$ each), bound antibodies were visualized using enhanced chemiluminescence ECL. $\beta$-actin was used as loading control.

Statistical analysis. Each experiment was repeated at least 3 times and confirmed that similar data were obtained. All data are expressed as mean and standard errors. Statistical significance was tested using one-way ANOVA with post Newman-Keuls test or two-way ANOVA with post Bonferroni test. Statistical differences were considered to be significant if $\mathrm{P}<0.05$.

\section{Results}

Nicotine treatment augments cell proliferation abilities in Raw264.7 and El4 cells. Our previous studies showed that nicotine could activate bone marrow-derived dendritic cells and nicotine-treated dendritic cells have potential antitumor effects (7-9). To explore the effect of nicotine on cell proliferation, both Raw264.7 and El4 cells were treated with $10^{-5}-10^{-8}$ $\mathrm{mol} / \mathrm{l}$ nicotine for $12 \mathrm{~h}$ or $10^{-7} \mathrm{~mol} / 1$ nicotine for indicated periods and cell viabilities, PCNA expression were determined by CCK- 8 assay and western blot analysis, respectively. The results showed that compared to PBS-treated control, $10^{-6}$ $10^{-8} \mathrm{~mol} / \mathrm{l}$ nicotine treatment clearly augmented Raw264.7 cell viability ${ }^{* * *} \mathrm{P}<0.001$, one-way ANOVA with post NewmanKeuls test) (Fig. 1A). Meanwhile, 16, 24 and 48 h $10^{-7} \mathrm{~mol} / \mathrm{l}$ nicotine treatment augmented Raw264.7 cell proliferation to $137.5,118.7$ and $126.7 \%$ respectively (Fig. 1C). PCNA protein determination also showed that nicotine treatment upregulated 
A

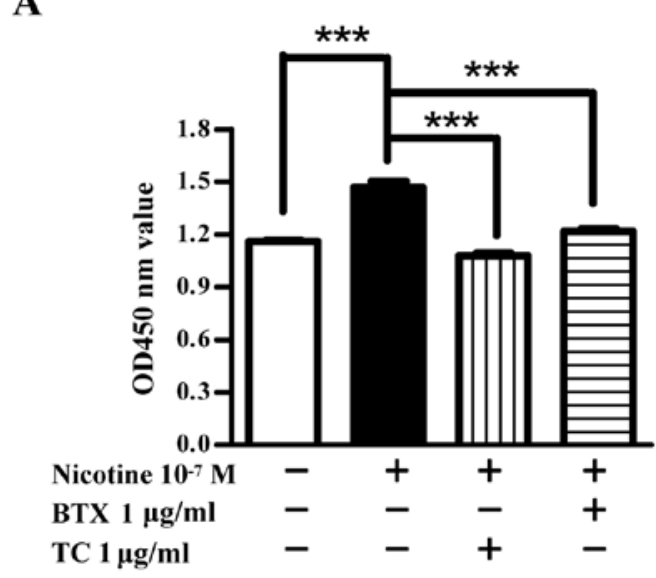

C

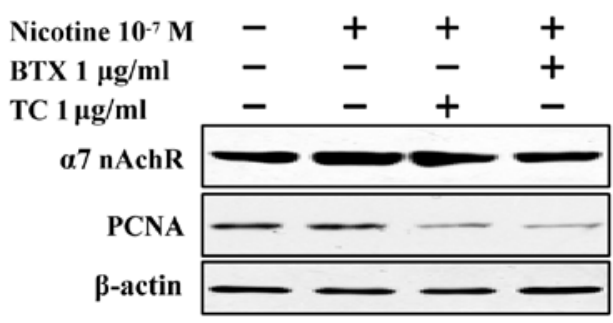

B

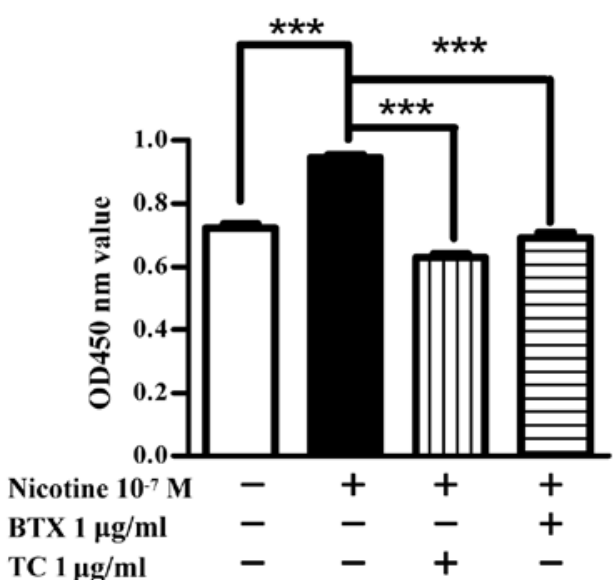

D

Figure 2. $\alpha 7 \mathrm{nAChR}$ is involved in nicotine-augmented cell proliferation in Raw264.7 and El4 cells. To explore the role of $\alpha 7 \mathrm{nAChR}$ in nicotine-augmented cell proliferation, Raw264.7 and E14 cells were pretreated with $1 \mu \mathrm{g} / \mathrm{ml}$ tubocurarine chloride or $\alpha$-bungarotoxin prior to $10^{-7}$ mol $/ 1$ nicotine stimulation. Cell viabilities of (A) Raw264.7 and (B) El4 cells were determined by CCK-8 assay. Data are given as mean \pm SEM, $n=4$. ${ }^{* * *}$ P $<0.001$, one way ANOVA with post Newman-Keuls test. PCNA and $\alpha 7 \mathrm{nAChR}$ expression of (C) Raw264.7 and (D) E14 cells was determined by western blot analysis. $\beta$-actin was used as internal control. A representative of three independent experiments is shown $(n=3)$. BTX, $\alpha$-bungarotoxin; TC, tubocurarine chloride.

PCNA expression in Raw264.7 cells (Fig. 1E). Cell viability and PCNA expression investigations in El4 cells presented similar results (Fig. 1B, D and F). These results illustrate that nicotine augments cell proliferation in both a concentration and period manner.

$\alpha 7 n A C h R$ is involved in nicotine-augmented cell proliferation in both Raw264.7 and El4 cells. $\alpha 7 \mathrm{nAChR}$, expressed in nonneuronal cells such as monocytes and endothelial cells, is one of the most abundant nicotinic acetylcholine receptors (11). To investigate the role of $\alpha 7 \mathrm{nAChR}$ in nicotine-increased cell proliferation, Raw264.7 and El4 cells were pretreated with $\alpha 7$ nAChR specific antagonist $\alpha$-bungarotoxin or non-specific antagonist tubocurarine chloride prior to nicotine treatment. Then, cell viability, $\alpha 7 \mathrm{nAChR}$ and PCNA expression were determined by CCK-8 assay and western blot analysis, respectively. The results showed that compared with vehicletreated cells, nicotine treatment significantly enhanced cell viability in both Raw264.7 and El4 cells (Fig. 2A and B). The pretreatment of $\alpha$-bungarotoxin or tubocurarine chloride clearly abolished the effect of nicotine on cell viability. For example, the pretreatment of $\alpha$-bungarotoxin or tubocurarine chloride achieved $\sim 83$ and $73 \%$ inhibitory rate, respectively, in Raw264.7 cells $\left({ }^{* * *} \mathrm{P}<0.001\right.$, one-way ANOVA with post Newman-Keuls test). As nicotine significantly upregulated $\alpha 7 \mathrm{nAChR}$ and PCNA expression in both Raw264.7 and El4 cells, the preincubation of $\alpha$-bungarotoxin or tubocurarine chloride abolished the effect of nicotine on $\alpha 7 \mathrm{nAChR}$ and PCNA expression (Fig. 2C and D). These data indicate that the upregulation of $\alpha 7 \mathrm{nAChR}$ induced by nicotine treatment contributes to nicotine-enhanced cell proliferation abilities.

Nicotine treatment upregulates anti-apoptotic protein Mcl-1 and Bcl-2 expression in Raw264.7 and El4 cells. To explore the effect of nicotine on anti-apoptotic protein $\mathrm{Mcl}-1$ and $\mathrm{Bcl}-2$ expression, Raw264.7 and El4 cells were treated with $10^{-7} \mathrm{M}$ nicotine for indicated periods, the expression of Mcl-1 and Bcl-2 was determined by western blot analysis. The results showed that the expression of Mcl-1 and Bcl-2 was continuously augmented by nicotine treatment in both Raw 264.7 (Fig. 3A) and El4 (Fig. 3B) cells, indicating that nicotine treatment might achieve anti-apoptotic effects on these cells.

$\alpha 7 n A C h R$ is involved in nicotine-inhibited cisplatin-induced caspase-3 activation in both Raw264.7 and El4 cells. To investigate the role of $\alpha 7 \mathrm{nAChR}$ in nicotine-mediated anti-apoptotic effects, Raw264.7 and El4 cells were treated with $\alpha$-bungarotoxin or tubocurarine chloride prior to nicotine stimulation, the cleaved caspase- 3 was determined by western blot analysis. The results showed that compared with cisplatin-treated cells, nicotine treatment significantly inhibited cisplatin-induced cleaved caspase- 3 in both Raw264.7 (Fig. 4A) and El4 (Fig. 4B) cells. Notably, the effect of nicotine on cisplatin-induced caspase-3 activation was efficiently abolished by the pretreatment of $\alpha$-bungarotoxin or tubocurarine chloride (Fig. 4), indicating that the upregulation of $\alpha 7 \mathrm{nAChR}$ induced by nicotine contributed to nicotine-mediated antiapoptotic effects. 
A

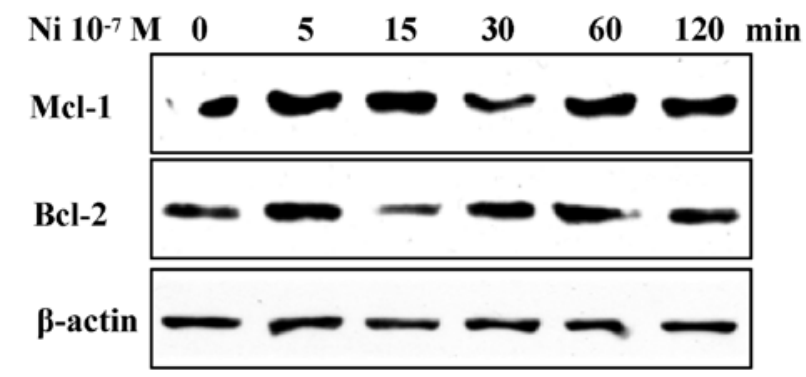

B

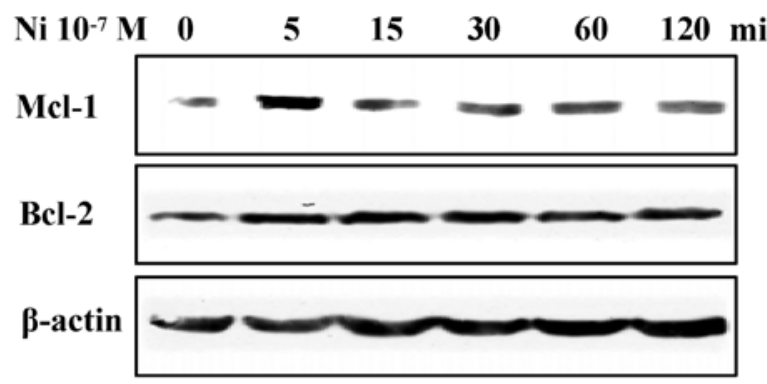

Figure 3. Nicotine treatment upregulates Mcl-1 and Bcl-2 expression in Raw264.7 and E14 cells. To explore the effects of nicotine on anti-apoptotic protein Mcl-1 and Bcl-2 expression, 2x10 (A) Raw264.7 and (B) El4 cells treated with $10^{-7} \mathrm{~mol} / 1$ nicotine for the indicated periods and whole cellular protein was extracted. The effect of nicotine on Mcl-1 or Bcl-2 expression in (A) Raw264.7 and (B) El4 cells was determined by western blot analysis. $\beta$-actin was used as internal control. A representative of three independent experiments is shown $(\mathrm{n}=3)$. Ni, nicotine.

A

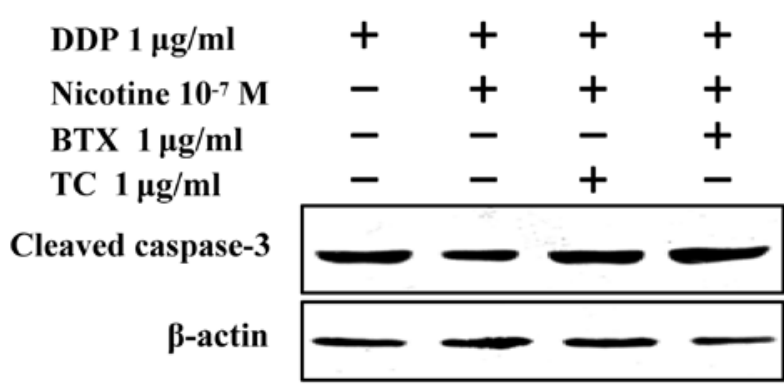

B

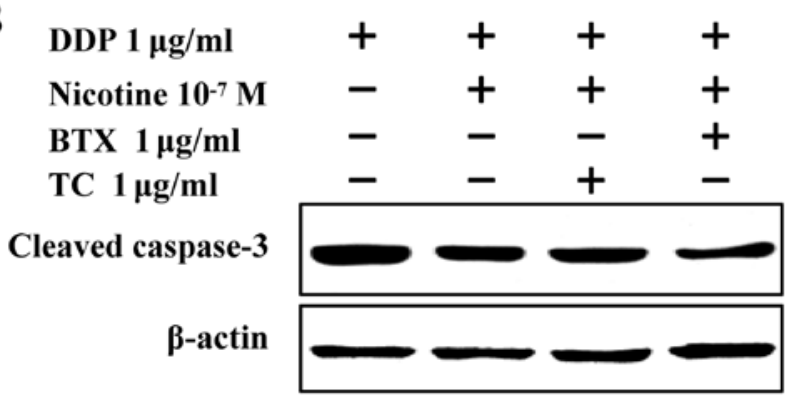

Figure 4. $\alpha 7 \mathrm{nAChR}$ is involved in nicotine-decreased cisplatin-induced caspase-3 activation in Raw264.7 and El4 cells. To explore the role of $\alpha 7 \mathrm{nAChR}$ in nicotine-mediated anti-apoptotic effect, $2 \times 10^{7}$ Raw 264.7 or El 4 cells were pretreated with tubocurarine chloride or $\alpha$-bungarotoxin prior to $10^{-7} \mathrm{~mol} / 1$ nicotine stimulation which was further treated with $1 \mu \mathrm{g} / \mathrm{ml}$ cisplatin. The cleaved caspase-3 of (A) Raw264.7 and (B) El4 cells was assessed by western blot analysis. A representative of three independent experiments is shown $(\mathrm{n}=3)$. $\beta$-actin was used as internal control. BTX, $\alpha$-bungarotoxin; TC, tubocurarine chloride; DDP, cisplatin.
A

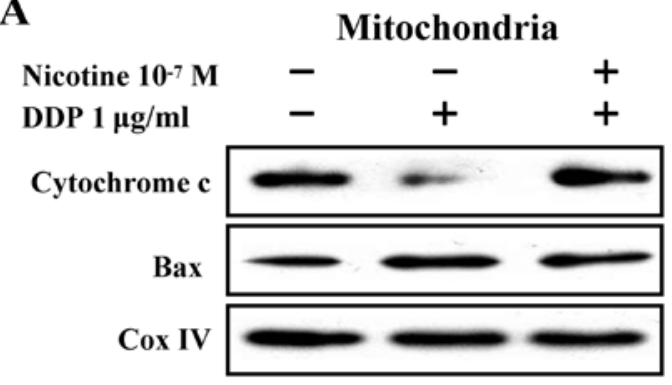

B

\section{Cytoplasm}

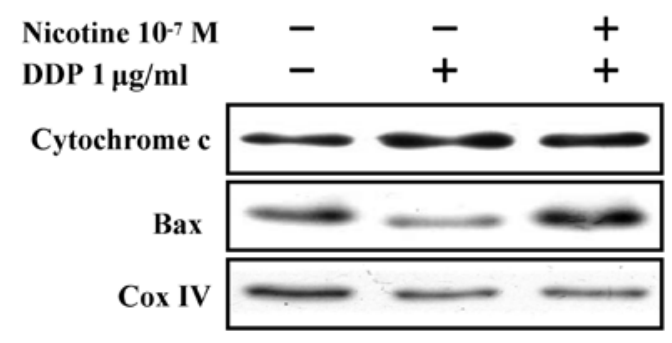

Figure 5. Nicotine attenuates cisplatin-induced mitochondrial translocation of Bax and cytochrome $c$ release in Raw264.7 cells. To investigate the effect of nicotine on cisplatin-induced mitochondrial translocation and cytochrome $c$ release, $2 \times 10^{7}$ Raw 264.7 cells were treated with $10^{-7} \mathrm{~mol} / 1$ nicotine prior to cisplatin $(1 \mu \mathrm{g} / \mathrm{ml})$ treatment. Then, (A) mitochondria and (B) cytosol fraction were extracted and the levels of Bax and cytochrome $c$ in both mitochondria and cytoplasm were determined by western blot analysis, respectively. A representative of three independent experiments is shown $(n=3) . \beta$-actin and Cox IV were used as cytoplasm and mitochondria internal control respectively. DDP, cisplatin.

The mitochondrial translocation of Bax is involved in nicotine-inhibited cisplatin-induced cytochrome c release in Raw264.7 cells. To investigate the role of Bax translocation in nicotine-mediated anti-apoptotic effects, Raw264.7 cells were stimulated with nicotine prior to cisplatin treatment and mitochondria/cytosol fraction was extracted. The mitochondrial translocation of Bax and the release of cytochrome $c$ were determined by western blot analysis. The results showed that the decreased cytochrome $c$ level and increased Bax existence in mitochondria was derived from cisplatin treatment (Fig. 5A). On the other hand, cisplatin stimulation was found to augment cytochrome $c$ level and decrease Bax expression in cytoplasm (Fig. 5B), indicating that mitochondrial translocation of Bax was involved in cisplatin-induced cytochrome $c$ release in Raw264.7 cells. The pretreatment of nicotine abolished cisplatin's effects on Bax translocation and cytochrome $c$ release (Fig. 5). Therefore, nicotine achieved anti-apoptotic effects by attenuating Bax translocation from cytoplasm to mitochondria.

Nicotine activates MAPK and PI3K-Akt pathways in both Raw264.7 and El4 cells. Erk1/2-p38-JNK and PI3K-Akt pathways are reported to be involved in regulating antiapoptosis protein expression (25-27) and promoting lung and colon cancer cell proliferation $(27,28)$, respectively. To investigate the roles of Erk1/2-p38-JNK and PI3K-Akt pathways in nicotine-mediated anti-apoptotic effects and promoting cell proliferation, Raw264.7 and El4 cells were treated with nicotine and the effects of nicotine on Erk1/2-p38-JNK and 
A

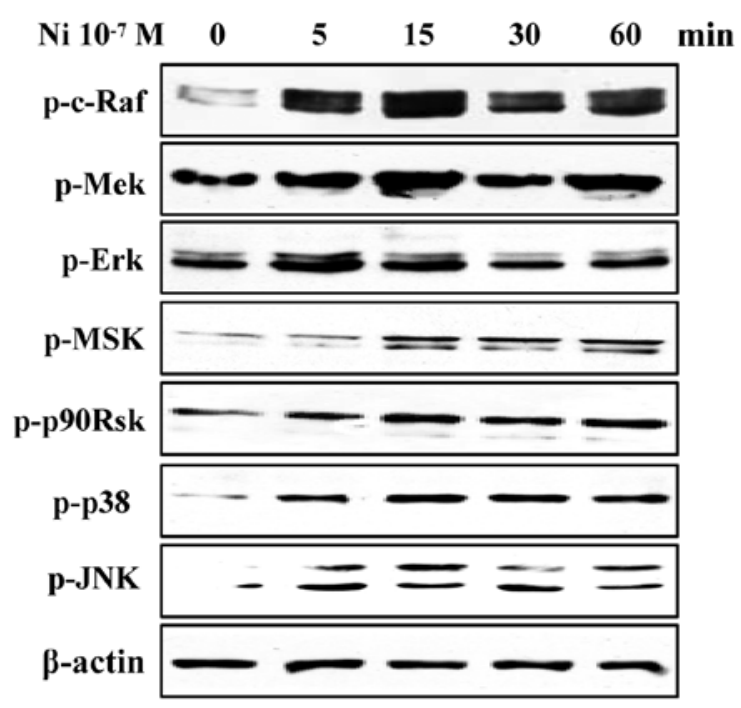

C

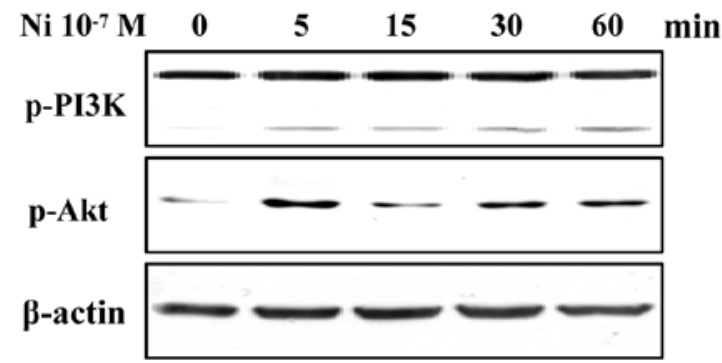

B

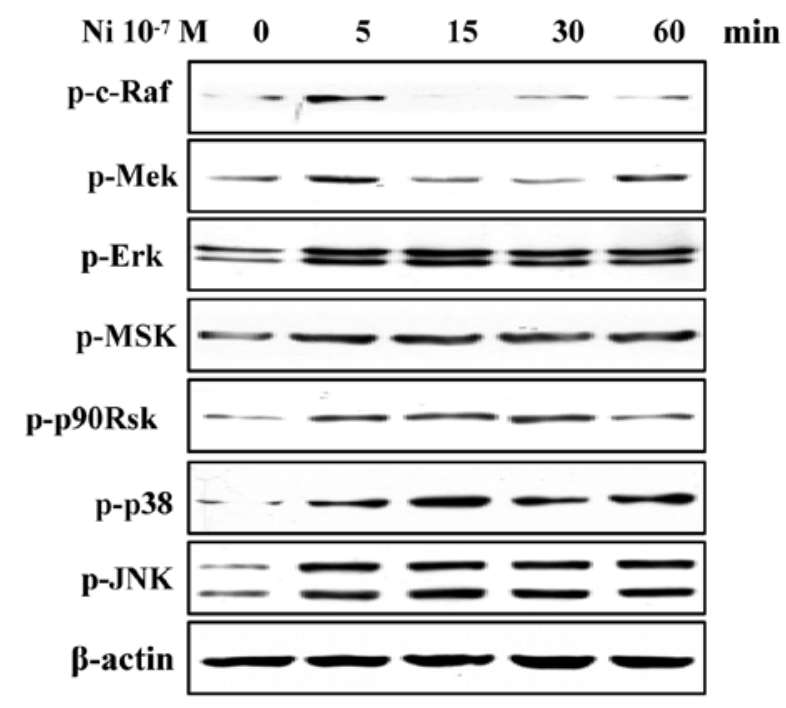

D

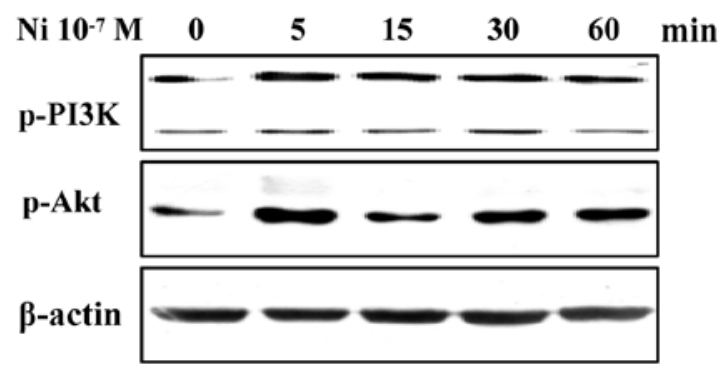

Figure 6. Nicotine efficiently activates Erk1/2-p38-JNK and PI3K-Akt pathways in both Raw264.7 and El4 cells. To determine effect of nicotine on MAPK and PI3K-Akt pathway activation, $2 \times 10^{7}$ Raw264.7 (A and C) or El4 (B and D) cells were treated with $10^{-7}$ mol/1 nicotine for the indicated periods and the phosphorylation of Erk1/2, MSK, p90rsk, Mek, c-raf, p38, SAPK/JNK (A and B) and PI3K, Akt (C and D) were determined by western blot analysis. $\beta$-actin was used as internal control. A representative of three independent experiments is shown $(\mathrm{n}=3)$. Ni, nicotine.

PI3K-Akt pathway activation was determined by western blot analysis. The results showed that Erk1/2-p38-JNK kinases and other components of these pathways, such as c-Raf, Mek, MSK and p90Rsk, were clearly activated by nicotine $\left(10^{-7} \mathrm{M}\right)$ treatment in both Raw264.7 (Fig. 6A) and El4 (Fig. 6B) cells. Meanwhile, the augmented phosphorylation levels of PI3K p55 (Tyr199), PI3K p85 (Tyr458) and Akt were visible within 5 min and continued to $60 \mathrm{~min}$ in both Raw264.7 (Fig. 6C) and El4 cells (Fig. 6D). These results indicate that nicotine treatment activated the MAPK and PI3K-Akt pathways.

The PI3K-Akt pathway is involved in nicotine-augmented cell proliferation. To investigate the role of the PI3K-Akt pathway in nicotine-augmented cell proliferation, Raw264.7 and El4 cells were pretreated with PI3K-Akt kinase inhibitor prior to nicotine stimulation and cell viabilities were determined by CCK- 8 assay. The results showed that while nicotine obviously augmented both Raw264.7 and E14 cell proliferation, the inhibition of PI3K kinase activities which was derived from LY294002 pretreatment achieved 36.6 and $44.9 \%$ inhibitory rates in Raw264.7 (Fig. 7A) and El4 (Fig. 7B) cells, respectively. Meanwhile, the usage of Wortmanin which is an inhibitor of Akt kinase also abolished the effects of nicotine on cell proliferation, which revealed 42.1 and $79.5 \%$ inhibitory rates in Raw264.7 (Fig. 7A) and El4 (Fig. 7B) cells, respectively $\left({ }^{* * *} \mathrm{P}<0.001\right.$, one-way ANOVA with post Newman-Keuls test) (Fig. 7). Hence, nicotine-induced PI3K-Akt activation is involved in the nicotine effects on cell proliferation.

The Erk-JNK pathway is involved in nicotine-mediated anti-apoptotic effects. To explore the role of Erk-p38-JNK MAPK pathway in nicotine-augmented anti-apoptotic effects, Raw264.7 and EL4 cells were pretreated with kinase inhibitors prior to nicotine stimulation and cell apoptosis was determined by flow cytometry. The results showed that while nicotine pretreatment decreased cisplatin-induced apoptosis from 51.2 to $33.8 \%$ and 24.5 to $12.6 \%$ in Raw264.7 (Fig. 8A) and El4 (Fig. 8B) cells, respectively, the usages of Erk kinase U0126 and JNK kinase SP600125 clearly abolished the effects of nicotine on cell anti-apoptosis in both Raw264.7 (Fig. 8A) and El4 (Fig. 8B) cells, respectively ${ }^{* * *} \mathrm{P}<0.001$, one-way ANOVA with post Newman-Keuls test) (Fig. 8). The inhibition of p38 kinase activities by the usage of SB203580 did not reverse nicotine's effects on cell apoptosis, indicating that p38 phosphorylation is not associated with nicotine's anti-apoptotic function. These results indicate that the Erk-JNK MAPK pathway is involved in nicotine-augmented anti-apoptotic effects. 
A

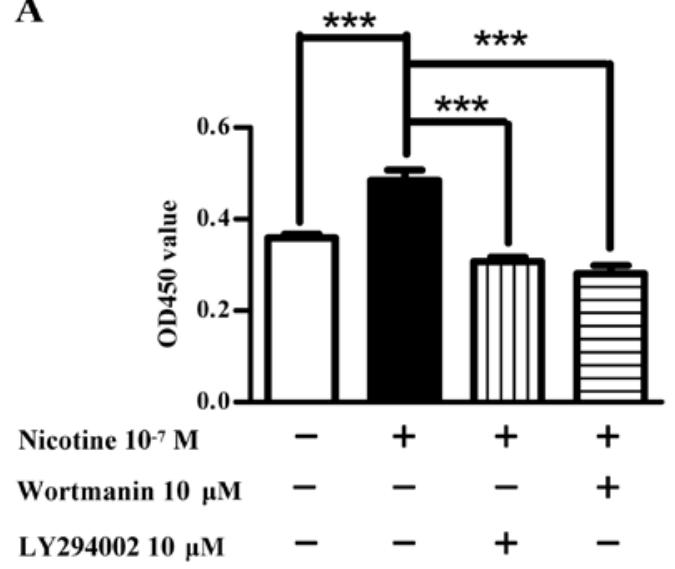

B

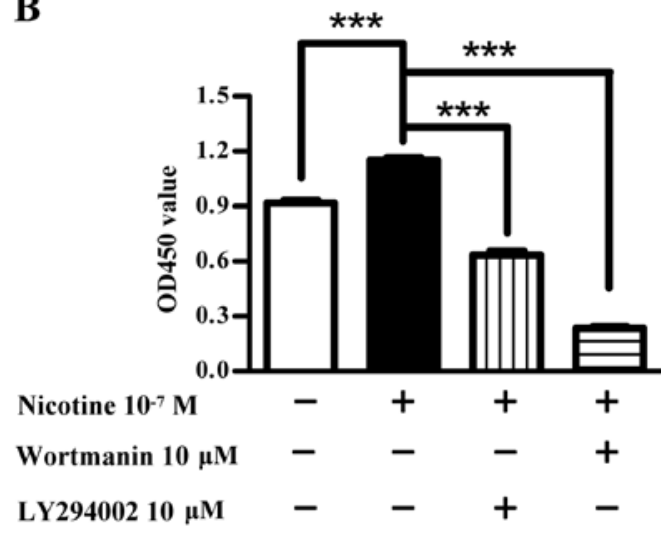

Figure 7. PI3K-Akt pathway activation is involved in nicotine-augmented cell proliferation in Raw264.7 and El4 cells. To explore the role of PI3K-Akt pathway in nicotine-augmented cell proliferation, $5 \times 10^{4}$ Raw264.7 or El4 cells were pretreated with $10 \mu \mathrm{M}$ LY294002 or Wortmanin prior to $10^{-7}$ mol/1 nicotine stimulation. Then, cell viabilities of (A) Raw264.7 or (B) El4 cells were determined by CCK-8 assay. Data are given as mean \pm SEM, $n=4$. ${ }^{* * *} \mathrm{P}<0.001$, one way ANOVA with post Newman-Keuls test. A representative of three independent experiments is shown $(\mathrm{n}=3)$.

A

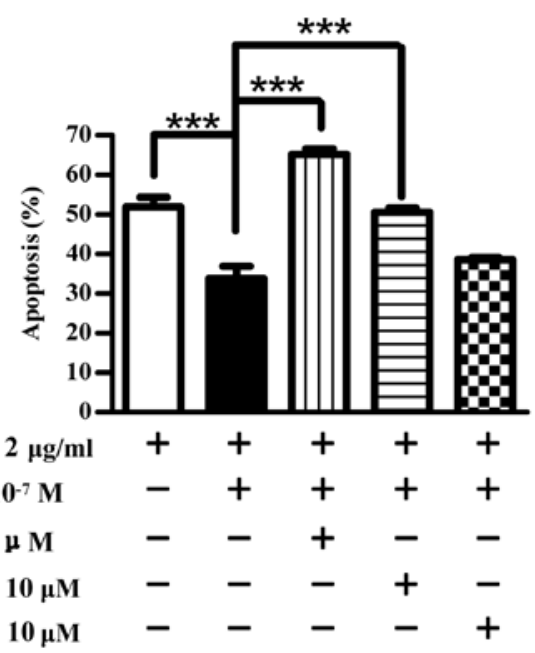

B

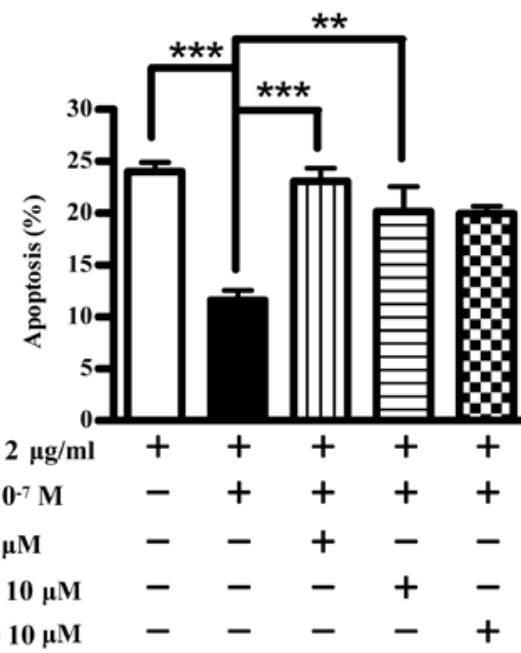

Figure 8. The phosphorylation of Erk1/2-JNK is responsible for nicotine-mediated anti-apoptotic effects in Raw264.7 and El4 cells. To explore the role of Erk1/2, JNK and p38 in nicotine-mediated anti-apoptotic effects, 5x10 ${ }^{4}$ Raw264.7 (A) or E14 (B) cells were pre-incubated with $10 \mu \mathrm{M}$ U0126, SB203580 or SP600125 prior to $10^{-7} \mathrm{~mol} / 1$ nicotine stimulation which were further treated with $2 \mu \mathrm{g} / \mathrm{ml}$ cisplatin. Then, cell apoptosis was assessed by flow cytometry with Annexin V/PI staining. Data are given as mean $\pm \mathrm{SEM}, \mathrm{n}=4 .{ }^{* * *} \mathrm{P}<0.001 ;{ }^{* *} \mathrm{P}<0.01$, one way ANOVA with post Newman-Keuls test. A representative of three independent experiments is shown $(\mathrm{n}=3)$.

\section{Discussion}

In recent years, our studies have focused on the effect of neurotransmitter on immune cells and we have found that nicotine, an acetylcholine agonist, could activate bone marrowderived dendritic cells and have potential antitumor effects (7-9). Further exploration showed that the biological effect of nicotine on lymphocyte is dependent on nicotine dose, duration of exposure and lipopolysaccharide existing in experiment system (23). However, other studies indicated that inhibition of $\alpha 7 \mathrm{nACh}$ mediated signaling could reduce tumorigenicity (18) and facilitate lung cancer treatment (19). Hence, the exact roles of $\alpha 7 \mathrm{nACh}$ in acetylcholine-mediated biological characterizations remain controversial and require further investigation. In the present study, Erk-JNK and PI3K-Akt signaling pathways were found to be respectively responsible for $\alpha 7 \mathrm{nACh}-$ mediated cell proliferation and anti-apoptotic effects which were supported by the following data; firstly, nicotine-augmented cell viabilities, upregulated $\alpha 7 \mathrm{nACh}$ and PCNA expression were obviously abrogated by the pretreatment of $\alpha 7 \mathrm{nAChR}$ antagonist $\alpha$-bungarotoxin and tubocurarine chloride; secondly, nicotine attenuated cisplatin-induced caspase- 3 activation and cytochrome $c$ release from mitochondria were reversed by the treatment of $\alpha$-bungarotoxin or tubocurarine chloride. Further studies showed that nicotine could efficiently activate the Erk-JNK-p38 and PI3K-Akt signaling pathway. The inhibition of Erk-JNK MAPK activities significantly decreased nicotinemediated anti-apoptotic effects. Of note, the downregulation of nicotine-augmented proliferation abilities was achieved by the usage of PI3K-Akt kinase inhibitor. 
AChRs, which is an integral membrane protein that responds to the binding of acetylcholine, can be classified as $\mathrm{nAChR}$ and $\mathrm{mAChR}$ according to their relative affinities and sensitivities to nicotine or muscarine. As nAChRs are widely expressed by nervous system (10) and non-neuronal cells (11), $\alpha 1, \alpha 3, \alpha 5 \mathrm{nAChRs}$ has been found to regulate cell proliferation and apoptosis (12-14). In the present study, the pretreatment of $\alpha 7 \mathrm{nAChR}$ specific antagonist $\alpha$-bungarotox in or non-specific antagonist tubocurarine chloride not only abrogated nicotine-augmented $\alpha 7 \mathrm{nAChR}$ upregulation, cell proliferation (Fig. 2) but also reversed nicotine-mediated anti-apoptotic effects (Fig. 4), indicating that $\alpha 7 \mathrm{nAChR}$ might be mainly acetylcholine receptor in Raw264.7 and El4 cells. However, it should be noted that $\alpha 7 \mathrm{nAChR}$, which was found to be involved in tumor migration $(15,16)$ and PPAR $\beta / \delta$ expression (17), has also been documented to facilitate dendritic cell-mediated antitumor immune response (7-9). Hence, the exact roles of $\alpha 7 \mathrm{nAChR}$ and other AChRs in nicotine-mediated proliferation and anti-apoptotic effects require further investigation.

Bcl-2 and Mcl-1, which belong to the Bcl-2 family, are located in the outer mitochondrial membrane and protect cells against a variety of apoptotic stimuli (29). In the present study, both Bcl-2 and Mcl-1 expression was upregulated by nicotine treatment in both Raw264.7 and El4 cells (Fig. 3). Hence, nicotine clearly inhibited cisplatin-induced mitochondrial dependent caspase-3 activation (Fig. 4) and cytochrome $c$ release (Fig. 5). The decreased Bax level in mitochondria and increased Bax level in cytoplasm induced by nicotine stimulation (Fig. 5) indicated that Bax translocation from cytoplasm to mitochondria might be a key step involved in nicotinemediated anti-apoptotic effects. Upon apoptotic signalling, Bak/Bax translocated to the mitochondrial outer membrane, inserted its transmembrane domain, oligomerized and released cytochrome $c$ (30). Cytochrome $c$ then binds to and penetrates lipid structures which contain the inner mitochondrial membrane lipid cardiolipin, leading to protein conformational changes and increased peroxidase activity (31). Hence, the exact mechanisms of Bax in nicotine decreased cytochrome $c$ release remain to be further examined.

Erk-JNK, one of the vital signal transduction pathways transmitting and converting stress signaling into apoptosis signaling (32), has been reported to upregulate Fas ligand expression (33) and is necessary for mitochondrial mediated apoptosis (34). Meanwhile, JNK activation playing an antiapoptotic function was also documented (35). In the present study, the inhibition of Erk-JNK kinase abrogated nicotinemediated anti-apoptotic effects in both Raw264.7 and El4 cells (Fig. 8), indicating that Erk-JNK phosphorylation facilitated cell survival in Raw264.7 and El4 cells. Although p38 could be efficiently activated (Fig. 6), the preincubation of SB203580 could not reverse nicotine-mediated anti-apoptotic effects, indicating that p38 phosphorylation is not associated with the anti-apoptotic function of nicotine.

Collectively, our study revealed that nicotine could achieve $\alpha 7$ nAChR-mediated proliferation effects by activating the PI3K-Akt pathway. Nicotine's anti-apoptotic effect might result from Bcl-2 family protein upregulation and Erk-JNK MAPK kinase phosphorylation, which provides potential molecules to deal with nicotine-associated human diseases.

\section{Acknowledgements}

This study was supported by grants from the National Natural Science Foundation of China (No. 81273203), the Natural Science Foundation of Xiamen (No. 3502Z20104002) and a grant from the National Laboratory for Oncogenes and Related Genes of China (90-08-02).

\section{References}

1. Printz C: Gap narrows in African American smoking-related cancers, increases in breast and colorectal cancers. Cancer 117: 2357, 2011.

2. Li S, Peng Q, Chen Y, You J, Chen Z, Deng Y, Lao X, Wu H, Qin X and Zeng Z: DNA repair gene XRCC1 polymorphisms, smoking, and bladder cancer risk: a meta-analysis. PLoS One 8: e73448, 2013

3. Albuquerque EX, Pereira EF, Alkondon M and Rogers SW: Mammalian nicotinic acetylcholine receptors: from structure to function. Physiol Rev 89: 73-120, 2009.

4. Cardinale A, Nastrucci C, Cesario A and Russo P: Nicotine: specific role in angiogenesis, proliferation and apoptosis. Crit Rev Toxicol 42: 68-89, 2012.

5. Tournier JM and Birembaut P: Nicotinic acetylcholine receptors and predisposition to lung cancer. Curr Opin Oncol 23: 83-87, 2011.

6. Guo L, Li L, Wang W, Pan Z, Zhou Q and Wu Z: Mitochondrial reactive oxygen species mediates nicotine-induced hypoxiainducible factor- $1 \alpha$ expression in human non-small cell lung cancer cells. Biochim Biophys Acta 1822: 852-861, 2012.

7. Jin HJ, Li HT, Sui HX, Xue MQ, Wang YN, Wang JX and Gao FG: Nicotine stimulated bone marrow-derived dendritic cells could augment HBV specific CTL priming by activating PI3K-Akt pathway. Immunol Lett 146: 40-49, 2012.

8. Jin HJ, Sui HX, Wang YN and Gao FG: Nicotine up-regulated 4-1BBL expression by activating Mek-PI3K pathway augments the efficacy of bone marrow-derived dendritic cell vaccination. $\mathrm{J}$ lin Immunol 33: 246-254, 2013.

9. Gao FG, Wan DF and Gu JR: Ex vivo nicotine stimulation augments the efficacy of therapeutic bone marrow-derived dendritic cell vaccination. Clin Cancer Res 13: 3706-3712, 2007.

10. Changeux JP: The nicotinic acetylcholine receptor: the founding father of the pentameric ligand-gated ion channel superfamily. J Biol Chem 287: 40207-40215, 2012.

11. Matteoli G and Boeckxstaens GE: The vagal innervation of the gut and immune homeostasis. Gut 62: 1214-1222, 2013.

12. Al-Wadei MH, Al-Wadei HA and Schuller HM: Pancreatic cancer cells and normal pancreatic duct epithelial cells express an autocrine catecholamine loop that is activated by nicotinic acetylcholine receptors $\alpha 3, \alpha 5$, and $\alpha 7$. Mol Cancer Res 10: 239-249, 2012.

13. Al-Wadei MH, Al-Wadei HA and Schuller HM: Effects of chronic nicotine on the autocrine regulation of pancreatic cancer cells and pancreatic duct epithelial cells by stimulatory and inhibitory neurotransmitters. Carcinogenesis 33: 1745-1753, 2012.

14. Wang S, Takayama K, Tanaka K, Takeshita M, Nakagaki N, Ijichi K, Li H and Nakanishi Y: Nicotine induces resistance to epidermal growth factor receptor tyrosine kinase inhibitor by a1 nicotinic acetylcholine receptor-mediated activation in PC9 cells. J Thorac Oncol 8: 719-725, 2013.

15. Lien YC, Wang W, Kuo LJ, Liu JJ, Wei PL, Ho YS, Ting WC, $\mathrm{Wu} \mathrm{CH}$ and Chang YJ: Nicotine promotes cell migration through alpha7 nicotinic acetylcholine receptor in gastric cancer cells. Ann Surg Oncol 18: 2671-2679, 2011.

16. Wei PL, Kuo LJ, Huang MT, Ting WC, Ho YS, Wang W, An J and Chang YJ: Nicotine enhances colon cancer cell migration by induction of fibronectin. Ann Surg Oncol 18: 1782-1790, 2011.

17. Sun X, Ritzenthaler JD, Zhong X, Zheng Y, Roman J and Han S: Nicotine stimulates PPAR $\beta / \delta$ expression in human lung carcinoma cells through activation of PI3K/mTOR and suppression of AP-2 $\alpha$. Cancer Res 69: 6445-6453, 2009.

18. Paleari L, Sessa F, Catassi A, Servent D, Mourier G, Doria-Miglietta G, Ognio E, Cilli M, Dominioni L, Paolucci M, Calcaterra A, Cesario A, Margaritora S, Granone P and Russo P: Inhibition of non-neuronal $\alpha 7$-nicotinic receptor reduces tumorigenicity in A549 NSCLC xenografts. Int J Cancer 125: 199-211, 2009. 
19. Paleari L, Negri E, Catassi A, Cilli M, Servent D, D'Angelillo R Cesario A, Russo P and Fini M: Inhibition of nonneuronal a7-nicotinic receptor for lung cancer treatment. Am J Respir Crit Care Med 179: 1141-1150, 2009.

20. Shi D, Guo W, Chen W, Fu L, Wang J, Tian Y, Xiao X, Kang T, Huang $\mathrm{W}$ and Deng W: Nicotine promotes proliferation of human nasopharyngeal carcinoma cells by regulating $\alpha 7 \mathrm{AChR}$, ERK, HIF-1 $\alpha$ and VEGF/PEDF signaling. PLoS One 7: e43898, 2012.

21. Cesario A, Russo P, Nastrucci C and Granone P: Is $\alpha 7-n A C h R$ a possible target for lung cancer and malignant pleural mesothelioma treatment? Curr Drug Targets 13: 688-694, 2012.

22. Brown KC, Lau JK, Dom AM, Witte TR, Luo H, Crabtree CM, Shah YH, Shiflett BS, Marcelo AJ, Proper NA, Hardman WE, Egleton RD, Chen YC, Mangiarua EI and Dasgupta P: MG624, an $\alpha 7-n A C h R$ antagonist, inhibits angiogenesis via the Egr-1/ FGF2 pathway. Angiogenesis 15: 99-114, 2012

23. Hu SX, Sui HX, Jin HJ, Ni XY, Liu XX, Xue MQ, Zhang Y and Gao FG: Lipopolysaccharide and dose of nicotine determine the effects of nicotine on murine bone marrow-derived dendritic cells. Mol Med Rep 5: 1005-1010, 2012.

24. Zhang SH and Huang Q: Etoposide induces apoptosis via the mitochondrial- and caspase-dependent pathways and in noncancer stem cells in Panc-1 pancreatic cancer cells. Oncol Rep 30: 2765-2770, 2013.

25. Park HY, Kim GY, Kwon TK, Hwang HJ, Kim ND, Yoo YH and Choi YH: Apoptosis induction of human leukemia U937 cells by 7,8-dihydroxyflavone hydrate through modulation of the Bcl-2 family of proteins and the MAPKs signaling pathway. Mutat Res 751: 101-108, 2013.

26. Zhu X, Jiang Y, Shan PF, Shen J, Liang QH, Cui RR, Liu Y, Liu GY, Wu SS, Lu Q, Xie H, Liu YS, Yuan LQ and Liao EY: Vaspin attenuates the apoptosis of human osteoblasts through ERK signaling pathway. Amino Acids 44: 961-968, 2013.
27. Chi J, Zhu Y, Fu Y, Liu Y, Zhang X, Han L, Yin X and Zhao D: Cyclosporin A induces apoptosis in H9c2 cardiomyoblast cells through calcium-sensing receptor-mediated activation of the ERK MAPK and p38 MAPK pathways. Mol Cell Biochem 367: 227-236, 2012.

28. Cucina A, Dinicola S, Coluccia P, Proietti S, D'Anselmi F, Pasqualato A and Bizzarri M: Nicotine stimulates proliferation and inhibits apoptosis in colon cancer cell lines through activation of survival pathways. J Surg Res 178: 233-241, 2012.

29. Kale J, Liu Q, Leber B and Andrews DW: Shedding light on apoptosis at subcellular membranes. Cell 151: 1179-1184, 2012.

30. Ferrer PE, Frederick P, Gulbis JM, Dewson G and Kluck RM: Translocation of a Bak C-terminus mutant from cytosol to mitochondria to mediate cytochrome $\mathrm{C}$ release: implications for Bak and Bax apoptotic function. PLoS One 7: e31510, 2012.

31. Bergstrom CL, Beales PA, Lv Y, Vanderlick TK and Groves JT: Cytochrome $\mathrm{c}$ causes pore formation in cardiolipin-containing membranes. Proc Natl Acad Sci USA 110: 6269-6274, 2013.

32. Li Y, Chen G, Zhao J, Nie X, Wan C, Liu J, Duan Z and Xu G: 2,3,7,8-Tetrachlorodibenzo-p-dioxin (TCDD) induces microglial nitric oxide production and subsequent rat primary cortical neuron apoptosis through $\mathrm{p} 38 / \mathrm{JNK}$ MAPK pathway. Toxicology 312: 132-141, 2013.

33. Davis RJ: Signal transduction by the JNK group of MAP kinases. Cell 103: 239-252, 2000.

34. Faris M, Kokot N, Latinis K, Kasibhatla S, Green DR, Koretzky GA and Nel A: The c-Jun N-terminal kinase cascade plays a role in stress-induced apoptosis in Jurkat cells by up-regulating Fas ligand expression. J Immunol 160: 134-144, 1998.

35. Minamino T, Christou H, Hsieh CM, Liu Y, Dhawan V, Abraham NG, Perrella MA, Mitsialis SA and Kourembanas S: Targeted expression of heme oxygenase-1 prevents the pulmonary inflammatory and vascular responses to hypoxia. Proc Natl Acad Sci USA 98: 8798-8803, 2001. 